\section{Limits of SEM Resolution}

David C. Joy

University of Tennessee \& Oak Ridge National Laboratory

Over the past decade the achievable resolution of the scanning electron microscope (SEM) in secondary electron (SE) imaging mode has improved by about one order of magnitude. In fact, instruments capable of demonstrating a resolution of one nanometer and exhibiting a probe size of less than $0.6 \mathrm{~nm}$ are not available. Continued improvements in electronoptics, electron sources, and in electronic and mechanical stability promise even smaller probe sizes that still contain adequate current for imaging. It is therefore relevant to consider what resolution might ultimately be achievable with an SEM in SE mode

The contrast detail in an SE image depends on two factors, the generation of secondaries, and their subsequent escape from the specimen. Changes in either, or both, of these factors will change the number of secondaries collected, and the spatial scale over which this change is accomplished will set the effective resolution of the image (assuming that the physical probe size is small enough to be neglected). At low and medium magnifications, i.e. below about $50 \mathrm{kx}$, the resolution is limited because the image detail is mostly supplied by the SE2 secondaries generated by backscattered electrons and the scale over which this signal changes is a significant fraction of the incident electron range ${ }^{1}$

At higher magnifications the image detail is carried by the SE1 secondaries, produced directly by the incident beam, while the SE2 contribution stays constant. The size of the SE1 generation volume is of the order of $1-3 \mathrm{~nm}$ in diameter at the full width half maximum value but perhaps ten times wider at the full width tenth maximum value, depending on the material, while the SE escape range is between 3 and $8 \mathrm{~nm}$. The image resolution is therefore determined by the escape of the SE thus, for example, each edge in the image will be visible as a bright fringe a few $\mathrm{nm}$ in width. The limit of SE imaging under these conditions clearly occurs when the dimension of the object becomes comparable to the SE escape range. The edges of the object are then no longer separable and the true shape and size of the feature cannot be observed.

To achieve a higher level of resolution than this "classical imaging limit" it is necessary to improve the localization of SE generation. For low atomic number and amorphous materials, this is most readily done by placing a thin $(<2 \mathrm{~nm})$, uniform, metal layer on the surface. Because the rate of $S E I$ production in a metal is much higher than that in an organic solid all of the SE1 generation occurs within the film, the magnitude of the SE1 signal increasing rapidly with the thickness of the layer. As shown in figure (1) this leads to the production of edge detail in the SE image with an edge width of the same order as the thickness of the metal. The definition of edge detail, and consequently the minimum feature size which can be imaged, can therefore be reduced by using a thinner metal coating layer. If the practical problems could be overcome then the limit to this procedure is reached when the film becomes too thin to act like a metal and produce

\section{Can Your X-ray Data Be Trusted?}

X-Ray Microanalysis on a SEM or TEM can produce good results, or bad data. How can you tell the difference? Use Electron Flight Simulator software for Windows to model the sample and show you where your $\mathrm{x}$-ray signal is coming from.

Visit our web site and download a demo copy: http://members.aol.com/smworld100/index.htm Elecinon Flight Sinmlator $2.0^{\mathrm{m}}$ Small World phone/fax: (415) 345-8013 a significant SE1 yield. Recent experimental data shows that this occurs when the film thickness is reduced to about $0.3 \mathrm{~nm}$, suggesting that the smallest feature that could be resolved would be of the order of $0.5 \mathrm{~nm}$ in width?

In crystalline metals and semiconductors the desired localization of SE production might be achieved by using the crystal lattice itself. The incident electron probe can be decomposed into a Bloch wave-field within the crystal. With an optimally chosen orientation of the beam to the lattice, placing the beam at an atom position will produce a wave-field with its anti-nodes on the atom centers. This will enhance SE generation since secondary electron production requires high angle inelastic scattering which will only occur close to the atom centers $^{3}$. The SE signal would then vary in intensity with the beam position with the periodicity of the crystal. Lattice imaging using secondary electrons might thus be a possibility provided that an adequate signal to noise ratio could be obtained. In either case the final limit to the resolution of the SE image will be the delocalization of SE1 production. The SE1 generation profile (figure 2) consists of two components. The narrow central region comes from SE generated by large angle inelastic scattering events, which the broader background comes from SE produced by small angle inelastic events ${ }^{3}$. A simple application of the Heisenberg uncertainly principle to give the diameter of localization $d_{\text {min }}$ for the production of an SE of energy $E_{S E}$ by an incident electron of energy $E_{0}$ gives

$$
d_{\min }=\frac{66 \sqrt{E_{0}}}{E_{s e}}
$$

which would suggest that at $25 \mathrm{keV}$, production of a $4 \mathrm{eV}$ secondary is delocalized over $70 \mathrm{~nm}$ or more. However, the value predicted by equation (1) applies only to the case of small angle scattering, corresponding to the minimum momentum transfer, and hence to the skirt of the SE1 distribution. The width of the central portion of the SE1 profile is limited by the delocalization associated with large angle inelastic scattering. In this case sufficient momentum must be transferred to the ejected free electron and equation (1) takes the form

$$
d_{\min }=\frac{0.2}{\sqrt{E_{s e}}} \mathrm{~nm}
$$

a result which is not independent of the incident energy and predicts a delocalization of only $0.1 \mathrm{~nm}$ for a $4 \mathrm{eV}$ electron ${ }^{4}$. It can thus be concluded that SE imaging can certainly be anticipated to resolutions below $0.4 \mathrm{~nm}$, using metal film to define SE production, and to even smaller limits in crystals. Delocalization might ultimately set a limit at around $1 \AA^{5}$.

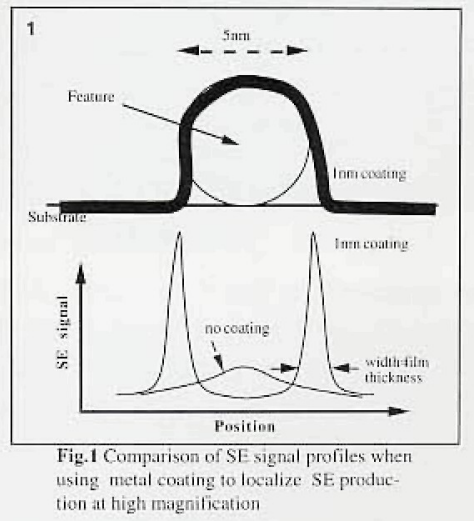

D.C. Joy, (1991), U/tramicroscopy 37,216

J. Liu et al, (1992), Proc 50th Ann. Meeting EMSA, 1288

J. Liu and J M. Cowley, (1988), Proc 46th. Ann. Meeting EMSA, 182

S.J. Pennycook, (1988), Ultramicroscopy 26, 239

Oak Ridge National Laboratory is managed by Martin Marietta Energy Systems Inc under contract DE-AC05-840R21400 with the U.S. Department of Energy

Reprinted from the 1994 Proceedings of the Microscopy Sociely of America 


\section{Get the Most from Your Imagel}

\section{The Ultimate}
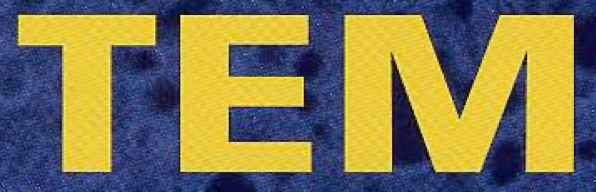

\section{Digital system}

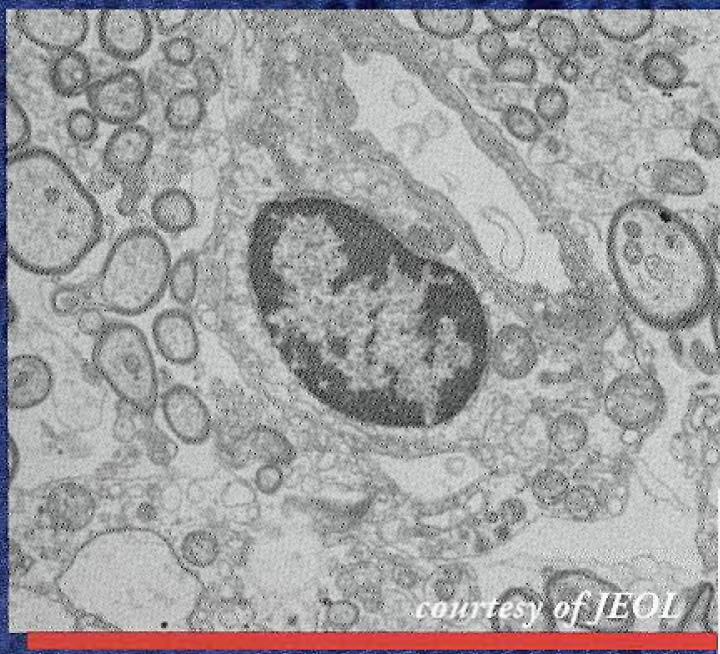

Highest Resolution

YAG Crystal Scintillator - Better

than the best phosphor

Up to $2 \mathrm{k} \times 3 \mathrm{k}$ camera resolution

Highest Sensitivity

Cooled CCD Camera

Ultra low noise electronics

\section{Easy to use}

Unique RS-170 output

PC or MAC versions \& even most popular workstations

Full support for your favorite 3rd party software

SURPRISINGIY AFFORDABLE!

\section{Princeton Instruments,inc.}

3660 Quakerbridge Road, Trenton, N.J. 08619

Tel: $609-587-9797$ Fax: $609-587-1970$

Email: sales@prinst.com World Wide Web: www prinst.com

Worldwide representation. Call or write for the representative nearest you. 